\title{
Germline TP53 c.566C > T mutation incidentally diagnosed during treatment for acute myeloid leukaemia: A case report
}

\author{
Hiroshi Sato ${ }^{1}$, Seiki Matsuo ${ }^{1}$, Yukiko Ando ${ }^{1}$, Yuko Imamura ${ }^{1}$, and Masaya Hirose ${ }^{1}$ \\ ${ }^{1}$ Hyogo Prefectural Amagasaki General Medical Center
}

November 3, 2021

\begin{abstract}
A 53-year-old woman was admitted at our hospital for the treatment of acute myeloid leukaemia. Heterozygous c.566C $>\mathrm{T}$ mutation in the TP53 gene was found in leukaemia cells and germline cells. The number of germline mutation found incidentally will increase. Therefore, patients with such incidental findings must undergo adequate genetic counselling.
\end{abstract}

\section{Introduction}

Li-Fraumeni syndrome is mainly caused by pathogenic germline mutations of the TP53 gene, most commonly missense mutations. Germline TP53c.566C $>$ T mutation results in the missense mutation at codon (A189V). This mutation has been observed in late-onset cancer such as multiple primary colon tumours, breast cancer and fallopian tube carcinoma.1-3 Moreover, it classified as a variant with uncertain significance. Recently, it was found not to be rare.4 However, the association between this mutation and haematological malignancy has been unclear. Herein, we report a case of germline TP53c.566C $>$ T mutation which was incidentally diagnosed during the treatment of acute myeloid leukaemia (AML).

\section{Case history/examination}

A 53-year-old Japanese woman presented with a 2-month history of general fatigue and vomiting. In addition, she experienced dizziness for 3 days and presented with a high fever on the admission day. She had no previous history of malignancy. Some of her family members developed late-onset cancers (Figure 1). Her mother (FII-3) had breast cancer at the age of 51 years. Her uncle (FII-5) developed stomach and colorectal cancer at the age of 60 and 62 years, respectively. Her grandmother (FI-4) died from colorectal cancer at the age of 62 years. She visited other hospitals for diagnosis and treatment. Examination results revealed leucocytosis (with a high number of blast cells), severe anaemia and thrombocytopenia. Computed tomography scan showed acute appendicitis and peritonitis. Hence, she was referred to our hospital for the treatment of AML and appendicitis.

The initial laboratory evaluation of the peripheral blood revealed the following: white blood cell count: $90.3 \times 10^{9} / \mathrm{L}(99 \%$ blast cells $)$, haemoglobin level: $4.5 \mathrm{~g} / \mathrm{dL}$, and platelet count: $5.0 \times 10^{9} / \mathrm{L}$. The patient's C-reactive protein level was elevated at $33.17 \mathrm{mg} / \mu \mathrm{l}$

Based on the pathologic examination, the bone marrow aspirate exhibited hypercellularity with $87 \%$ of blast cells. Chromosome analysis of leukaemia cells revealed a complex karyotype (46, XX, add(4)(q21), $\operatorname{add}(10)(\mathrm{p} 11.2)[9] / 46$, idem, add(14)(q22)[3]/46, XX [3]). Approximately $87 \%$ of the leukaemia cell population tested positive for CD34, HLADR, myelopeloxydasemyeloperoxidase and CD33. Meanwhile, the blast cell population tested negative for CD13 and CD38.

\section{Differential diagnosis, investigations and treatment}


Appendectomy and abdominal lavage were performed 2 days after admission. The initial chemotherapy was delayed for 7 days to allow for adequate wound healing postoperatively. The patient received induction chemotherapy for AML (aclarubicin $14 \mathrm{mg} / \mathrm{m}^{2}$ for 4 days, cytarabine $20 \mathrm{mg} / \mathrm{m}^{2}$ for 14 days and granulocyte colony-stimulating factor [CAG regimen]). Haematological complete remission was achieved after four cycles of chemotherapy. However, $1 \%$ of leukaemia cells remained in the bone marrow, which was confirmed via a flow cytometry analysis. Azacitidine $\left(75 \mathrm{mg} / \mathrm{m}^{2}\right.$ for 7 days) and venetoclax (50 mg/body weight daily) regimen was provided as a maintenance therapy. According to the National Comprehensive Cancer Network guidelines, this patient had a poor prognosis. Haematopoietic stem cell transplantation was planned. After two courses of azacitidine-venetoclax regimen, the patient received umbilical cord blood transplantation.

Somatic TP53 mutations of the AML cells were examined to predict prognosis. Heterozygous c.566C $>\mathrm{T}$ mutation was found in AML cells (Figure 2A). Because germline TP53 mutation was suspected, the haematologist referred the patient to the department of clinical genetics in our hospital. Analysis of the peripheral blood revealed a germline heterozygotic c.566C $>$ T mutation in the AML specimen (Figure 2B). The analysis was performed after the achievement of complete remission and before umbilical cord transplantation.

\section{Outcome and follow-up}

We recommended that her two daughters (FIV-1, aged 24 years and FIV-2, aged 20 years) undergo genetic counselling.

\section{Discussion}

The current case report aimed to provide oncologists with information about possible incidental findings including germline mutation.

TP53 is an extremely important tumour suppressor gene. Germline mutation in the TP53 gene causes Li-Fraumeni syndrome, a rare hereditary cancer syndrome. Somatic deleterious mutation in both alleles of the TP53 gene is commonly observed in sporadic cancer.TP53 mutations, which are detected in only 5\%$10 \%$ of AML cases, are associated with complex cytogenetic abnormalities and poor outcomes. AML with a complex karyotype accounts for 10\%-15\% of all adult AML cases. TP53somatic mutation was identified in about $60 \%$ of patients with AML patients who had a complex karyotype. 5 According to this report,TP53 mutation is strongly correlated with a complex karyotype.

AML associated with TP53 mutation is associated with a poor response to chemotherapy and short overall survival. Moreover, it is highly associated with therapy-related AML. However, our patient did not have a previous history of malignancy. Treatment with TP53mutation has been challenging in patients with AML. Nevertheless, induction chemotherapy was more challenging because leukaemia had been complicated by severe infection including appendicitis. Wei et al. showed that CAG regimen was effective and safe for patients with AML, and it may be more effective than non-CAG regimens.6

A new therapeutic strategy, including epigenetic drugs, has been found to be effective in targeting TP53 -mutated AML. Venetoclax is a bcl-2 inhibitor, and azacitidine is a hypomethylating agent.7 Venetoclax combined with hypomethylating agents including azacitidine has a promising therapeutic effect. Apoptosis mediated by venetoclax may be independent of TP53. Combination therapy with these drugs has been recently approved for the treatment of AML by the National Health Insurance of Japan. Azacitidinevenetoclax therapy can be used as a maintenance therapy. In our case, the TP53c.566C $>\mathrm{T}$ mutation in AML cells was heterozygous. Nevertheless, no other TP53 mutations were detected. If one allele of the TP53 gene was a wild type and had a normal function, the prognosis of this patient might have been better than that of patients with homozygous or compound heterozygous mutation. However, we cannot rule out the risk of loss of function without mutation including epigenetic silencing. TP53 mutations occur in $8 \%$ of de novo AML cases. In other solid malignancies, TP53 mutation is found in more than half of cases. TP53 abnormalities without mutation occur more frequently than expected.8 Several mechanisms underlying TP53 dysfunction have been reported. An algorithm for the TP53 -based diagnostic workup and treatment of AML was developed. Hence, the mutated or nonmutated disfunction of TP53must be identified for the 
treatment of AML.

Based on data in ClinVar, TP53c.566C $>\mathrm{T}$ mutation was considered a variant with uncertain significance. To the best of our knowledge, three cases with germline TP53 c.566C $>\mathrm{T}$ mutation have been reported in detail.1-3 No case has met the classical criteria of Li-Fraumeni syndrome. Miyaki et al.1 reported a case of multiple colorectal cancer in a 73-year-old man, and examination results showed that tumour cells had a compound heterozygous mutation. Cho et al.2 presented a case of unilateral breast cancer in a 47 -year-old woman, and assessment findings revealed that tumour cells had a homozygous mutation. The patient's father and brother had oesophageal and lung cancer, respectively. Her offspring underwent genetic counselling and genetic testing. Choi et al.3 showed a case of fallopian tube cancer in a 56-year-old patient who had germline pathogenic mutation in the BRCA1 gene. The patient's sister had ovarian cancer. TP53 mutation c.566C $>\mathrm{T}$ may be found incidentally. However, there can be no association between this mutation and carcinogenesis. In our case, leukaemia cells only exhibited heterozygous mutation. New somatic mutation did not occur in the TP53 gene of leukaemia cells. However, loss of function without mutation cannot be ruled out. Not only TP53 mutation but also epigenetic deregulation of TP53 expression is responsible for clinical heterogeneity.9 It was challenging to perform appropriate genetic counselling and surveillance on the patient and her family. Based on the family history, some members developed and colorectal cancer at $>50$ years of age. In previously reported cases, colorectal cancer was diagnosed at the age of 73 years old and breast cancer at the age of 47 years. This mutation may be correlated with relatively late-onset cancer. According to the family history of our patient and previous case reports, annual screening for breast and colorectal cancer may be recommended if a patient has germline TP53 c.566C $>\mathrm{T}$ mutation. Momozawa et al.4 showed that TP53 c.566C $>$ T mutation was found in 19 of 7051 women with breast cancer. Moreover, they revealed that the mutation was found in 17 of 11241 controls in a Japanese population. The odds ratio was 1.8 (0.9-3.7), and the $\mathrm{P}$ value was 0.088 . Hence, the results between the two groups did not significantly differ. About 1 in 660 healthy women had the mutation, which may not be a pathogenic variant for Li-Fraumeni syndrome or Li-Fraumeni-like syndrome. The association between the mutation and breast cancer can possibly be weak. In AML or other malignancies, the effect of the mutation was unclear. In the current case, the patient was informed about the mutation. Hence, her offspring was recommended to undergo genetic counselling, and cancer screening must be discussed. Moreover, the patient and her family should be informed that future studies may change the current interpretation of the mutation.

Several companion diagnostic tests, which are used to assess a specific biomarker for qualifying a patient to receive a specific, associated therapy, have been applied in clinical settings. Most of them are somatic and/or germline genetic tests. In Japan, two gene panel tests for genomic profiling have recently been covered by the National Health Insurance. In other countries, the number of patients who undergo such gene panel tests is increasing. Hence, the number of germline mutation found incidentally will also increase. It is more challenging to interpret VUS found incidentally than pathogenic mutation. Therefore, patients with such incidental findings should undergo adequate genetic counselling.

\section{Author Contributions}

HS, SM and MH designed the research study. HS and YI performed the research. TH and HS analyzed the data. HS, YA and MH wrote the manuscript. All authors contributed to editorial changes in the manuscript. All authors read and approved the final manuscript.

\section{Acknowledgements}

Written informed consent was obtained from the patient for publication of this case report and accompanying images.

\section{Conflict of Interest Statement}

All authors declare that they have no conflict of interest.

\section{Data Availability Statement}


The data that support the findings of this study are available from the corresponding author upon reasonable request.

\section{References}

1. Miyaki M, Iijima T, Ohue M, et al. A novel case with germline p53 gene mutation having concurrent multiple primary colon tumours. Gut 2003;52(2):304-306. doi:10.1136/gut.52.2.304.

2. Cho Y, Kim J, Kim Y, Jeong J, Lee KA. A case of late-onset Li-Fraumeni-like syndrome with unilateral breast cancer. Ann Lab Med 2013;33(3):212-216. doi:10.3343/alm.2013.33.3.212.

3. Choi MC, Bae JS, Jung SG, et al. Prevalence of germline BRCA mutations among women with carcinoma of the peritoneum or Fallopian tube. J Gynecol Oncol 2018 Jul;29(4):e43. doi:10.3802/jgo.2018.29.e43. Epub 2018 Mar 26.PMID:29770616.

4. Momozawa Y, Iwasaki Y, Parsons MT, et al. Germline pathogenic variants of 11 breast cancer genes in 7,051 Japanese patients and 11,241 controls. Nat Commun 2018 Oct 4;9(1):4083. doi:10.1038/s41467-018$06581-8$.

5. Rücker FG, Schlenk RF, Bullinger L, et al. TP53 alterations in acute myeloid leukemia with complex karyotype correlate with specific copy number alterations, monosomal karyotype, and dismal outcome. Blood 2012 Mar 1;119(9):2114-2121. doi:10.1182/blood-2011-08-375758. Epub 2011 Dec 20.

6. Wei G, Ni W, Chiao JW, Cai Z, Huang H, Liu D. A meta-analysis of CAG (cytarabine, aclarubicin, GCSF) regimen for the treatment of 1029 patients with acute myeloid leukemia and myelodysplastic syndrome. J Hematol Oncol 2011 Nov 14;4:46. doi:10.1186/1756-8722-4-46.

7. Nechiporuk T, Kurtz SE, Nikolova O, et al. The TP53 apoptotic network is a primary mediator of resistance to BCL2 inhibition in AML cells. Cancer Discov 2019 ;9(7):910-925. doi:10.1158/2159-8290.CD19-0125. Epub 2019 May 2.

8. Prokocimer M, Molchadsky A, Rotter V. Dysfunctional diversity of p53 proteins in adult acute myeloid leukemia: projections on diagnostic workup and therapy. Blood 2017;130(6):699-712. doi:10.1182/blood-201702-763086. Epub 2017 Jun 12.

9. Gargallo P, Yáñez Y, Segura V, et al. Li-Fraumeni syndrome heterogeneity. Clin Transl Oncol 2020;22(7):978-988. doi:10.1007/s12094-019-02236-2. Epub 2019 Nov 5.

\section{Figure legends}

Figure 1: Pedigree of the patient and her family. Generations are in Roman numerals. Individuals are in Arabic numbers. The solid symbol indicates individuals diagnosed with any type of cancer. The proband is marked by an arrow and $\mathrm{P}$ character.

Figure 2: Nucleotide sequence analysis of the TP53 gene including the coding sequence 566 (arrow). Leukaemia cells had a heterozygotic c.566C $>\mathrm{T}$ mutation $(2 \mathrm{~A})$. Analysis of the peripheral blood revealed a germline heterozygotic c.566C $>\mathrm{T}$ mutation (2B). 


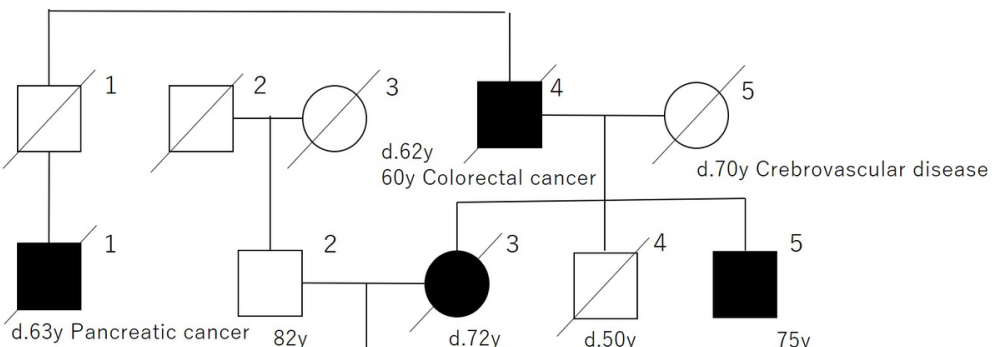

III

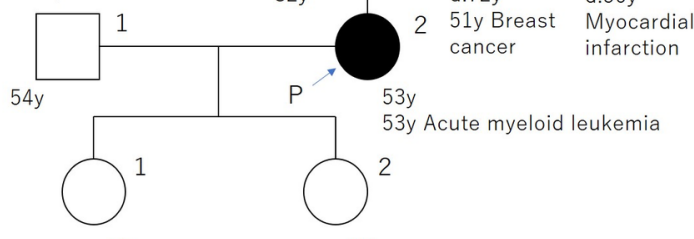

$75 y$

$24 y$

$20 y$
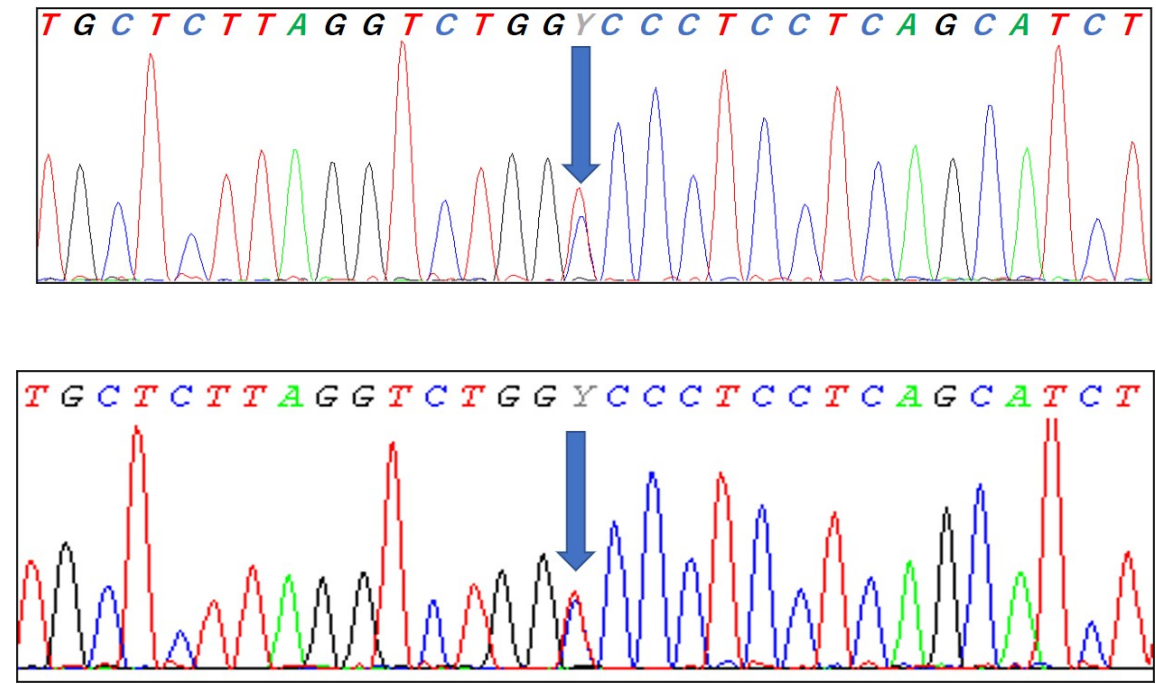